\title{
Vasopressin regulates the growth of the biliary epithelium in polycystic liver disease
}

\author{
Romina Mancinelli ${ }^{1}$, Antonio Franchitto ${ }^{1,2}$, Shannon Glaser ${ }^{3,4,5}$, Antonella Vetuschi ${ }^{6}$, Julie Venter ${ }^{3,4,5}$, Roberta Sferra ${ }^{5}$, \\ Luigi Pannarale ${ }^{1}$, Francesca Olivero ${ }^{1}$, Guido Carpino', Gianfranco Alpini ${ }^{3,4,5}$, Paolo Onori ${ }^{1}$ and Eugenio Gaudio ${ }^{1}$
}

The neurohypophysial hormone arginine vasopressin (AVP) acts by three distinct receptor subtypes: V1a, V1b, and V2. In the liver, AVP is involved in ureogenesis, glycogenolysis, neoglucogenesis and regeneration. No data exist about the presence of AVP in the biliary epithelium. Cholangiocytes are the target cells in a number of animal models of cholestasis, including bile duct ligation (BDL), and in several human pathologies, such as polycystic liver disease characterized by the presence of cysts that bud from the biliary epithelium. In vivo, liver fragments from normal and BDL mice and rats as well as liver samples from normal and ADPKD patients were collected to evaluate: (i) intrahepatic bile duct mass by immunohistochemistry for cytokeratin-19; and (ii) expression of V1a, V1b and V2 by immunohistochemistry, immunofluorescence and real-time PCR. In vitro, small and large mouse cholangiocytes, H69 (non-malignant human cholangiocytes) and LCDE (human cholangiocytes from the cystic epithelium) were stimulated with vasopressin in the absence/presence of AVP antagonists such as OPC-31260 and Tolvaptan, before assessing cellular growth by MTT assay and CAMP levels. Cholangiocytes express V2 receptor that was upregulated following BDL and in ADPKD liver samples. Administration of AVP increased proliferation and CAMP levels of small cholangiocytes and LCDE cells. We found no effect in the proliferation of large mouse cholangiocytes and H69 cells. Increases were blocked by preincubation with the AVP antagonists. These results showed that AVP and its receptors may be important in the modulation of the proliferation rate of the biliary epithelium.

Laboratory Investigation (2016) 96, 1147-1155; doi:10.1038/labinvest.2016.93; published online 29 August 2016

Arginine vasopressin, ${ }^{1}$ also known as antidiuretic hormone, ${ }^{1}$ is a neurohypophysial hormone having multiple functions, including renal water reabsorption, cardiovascular homeostasis and hormone secretion from the anterior pituitary. ${ }^{2,3}$ AVP is produced by neurons in the paraventricular and supraoptic nuclei of the hypothalamus. ${ }^{4,5}$ The hormone is released upon an increase in extracellular fluid osmolarity or a decrease in blood pressure and acts on target organs through several receptors: V1a, V1b and V2, that are expressed in different target organs and mediate distinct effects. ${ }^{4} \mathrm{Vla}$ is expressed predominantly in the walls of arteries, arterioles and veins and mediates vasoconstriction. It also increases renin production by acting on renal granule cells, indirectly increasing angiotensin II and aldosterone. ${ }^{6} \mathrm{~V} 1 \mathrm{~b}$ (or V3) is expressed by anterior pituitary cells and stimulates the release of adreno cortico tropic hormone. ${ }^{7} \mathrm{~V} 1 \mathrm{a}$ and $\mathrm{V} 1 \mathrm{~b}$ are also expressed by chromaffin cells of the adrenal medulla where they stimulate catecholamine secretion. ${ }^{8} \mathrm{~V} 2$ is found in the distal tubules and collecting ducts of the kidneys and mediates water reabsorption. ${ }^{9}$ The three AVP receptors are G-protein coupled receptors. Upon ligand binding, the cellular levels of second messengers (such as cAMP, $\mathrm{Ca}^{2+}$, inositol 1,4,5triphosphate and diacylglycerol) increase. ${ }^{10} \mathrm{~V} 2$ receptor is associated with a $G_{s}$ protein and, upon activation, cAMP levels increase; this causes the translocation of aquaporin2 and the amiloride-sensitive epithelial $\mathrm{Na}^{+}$channel in the principal cells of the collecting duct. ${ }^{11}$ In addition to regulating water and electrolyte balance and vasoconstriction, AVP also affects the promotion of cellular growth and proliferation, lipid metabolism and protein turnover. ${ }^{12}$ Cell proliferation and growth were observed in several types of cells: fibroblasts, vascular endothelial and smooth muscle

\footnotetext{
'Department of Anatomical, Histological, Forensic Medicine and Orthopedics Sciences, "Sapienza" University of Rome, Rome, Italy; ${ }^{2}$ Eleonora Lorillard Spencer Cenci Foundation, Rome, Italy: ${ }^{3}$ Department of Research, Central Texas Veterans Health Care System, Temple, TX, USA: ${ }^{4}$ Baylor Scott \& White Digestive Disease Research Center, Baylor Scott \& White, Temple, TX, USA; ${ }^{5}$ Department of Medicine, Division Gastroenterology, Texas A\&M University Health Science Center, Temple, TX, USA and ${ }^{6}$ Department of Biotechnological and Applied Clinical Sciences, University of L'Aquila, L'Aquila, Italy

Correspondence: Professor P Onori, MD, Department of Anatomical, Histological, Forensic Medicine and Orthopedics Sciences, "Sapienza" University of Rome, Via A. Borelli, 50, Rome 00161, Italy.

E-mail: paolo.onori@uniroma1.it

Received 16 May 2016; revised 19 July 2016; accepted 25 July 2016
} 
cells, cardiomyocytes, and also hepatocytes. ${ }^{13}$ Moreover, in human pathology, vasopressin is also involved in autosomaldominant polycystic kidney disease (ADPKD), a condition characterized by bilateral renal cysts, kidney pain, hypertension and progressive loss of renal function, owing to mutations in plasma membrane-spanning polycystin 1 and 2 (PKD1 and PKD2, respectively) genes. ${ }^{14}$ In many patients with ADPKD, there is the development of hepatic cysts, which originate from cholangiocytes, the cells that form the biliary epithelium, linked to the dysfunction of the primary cilium. ${ }^{15-17}$ Primary cilia have been identified as key organelles in the pathogenesis of ADPKD and related cystic diseases. ${ }^{18}$ In the liver parenchyma, only cholangiocytes possess primary cilia. They are regarded as the antennas of a cell, involved in mechano-, osmo-, and chemo-reception of bile flow and composition. ${ }^{19}$ The ciliary compartment is also the active site of the protein products of hepatocystic disease genes, such as PKD1 and PKD2. ${ }^{18}$ Liver cysts are usually asymptomatic, but the progressive growth may cause dyspnea, gastroesophageal reflux and nausea and mechanical low back pain arise because of the mass effect of the polycystic liver. ${ }^{20}$ Recently, the somatostatin analogs lanreotide and octreotide plus mTOR inhibitors have represented promising new agents for the treatment of patients with PLD. ${ }^{21,22}$ These agents reduce intracellular levels of cAMP by activating signaling cascades through the $\mathrm{Gi}_{\alpha}$ subunit, thereby preventing accumulation of fluid in liver cysts. ${ }^{23}$ AVP has a role in the pathogenesis of renal cysts in ADPKD, stimulating the cyst growth in several rodent models and in cultured human cells. ${ }^{24}$ Currently, there are no data on the effects of vasopressin and its receptor antagonists in the growth of hepatic cysts during PLD. For this reason, our aim has been to evaluate the role and the effect of the hormone in the growth of the biliary epithelium.

\section{MATERIALS AND METHODS Materials}

All reagents were purchased from Sigma-Aldrich (St Louis, MO, USA) unless otherwise indicated. The V2 primers for real-time PCR were purchased from SABiosciences (Frederick, MD, USA). The RNeasy Mini Kit to purify total cholangiocyte RNA was purchased from Qiagen (Valencia, CA, USA). The EIA kits for assessing cell proliferation were purchased from Cayman Chemical (Ann Arbor, MI). All the antibodies were purchased from Santa Cruz Biotechnology (Santa Cruz, CA, USA) or Sigma-Aldrich.

\section{Rat and Mouse Liver Samples}

Male Fischer 344 rats and male C57BL/6 mice were purchased from Charles River (Wilmington, MA, USA), maintained in a temperature-controlled environment $\left(20-22^{\circ} \mathrm{C}\right)$ with a 12 -h light-dark cycle and fed ad libitum standard chow. Animals had free access to drinking water. Our studies were carried out in normal and 1-week bile duct ligated (BDL) rats or mice. ${ }^{25}$ Before each experimental procedure, the animals were anesthetized by sodium pentobarbital $(50 \mathrm{mg} / \mathrm{kg}$ body wt i.p.) following the regulations of the panel on euthanasia of the American Veterinarian Medical Association and local authorities. All protocols were approved by the Institutional Animal Care and Use Committee of Baylor Scott \& White, Temple, TX, USA.

\section{Human Liver Samples}

We studied eight liver specimens obtained for diagnostic purposes at surgery with a diagnosis of ADPKD. Liver cysts were subdivided on the basis of their size as large $(>3 \mathrm{~cm}$ maximum diameter) or small cysts $(<3 \mathrm{~cm}$ maximum diameter). As controls, we evaluated liver biopsies with a normal histology from patients submitted to laparotomy (four fragments). The procedures followed for this study were in accordance with the institutional committee and abided by the ethical guidelines of the 1975 Declaration of Helsinki.

\section{Immunohistochemistry}

Immunohistochemistry was performed in 3- $\mu \mathrm{m}$ sections from liver fragments of rat, mouse and human. Sections were deparaffinized, then hydrated and rinsed in $1 \times$ phosphatebuffered saline (PBS, pH 7.4) before applying the selected primary antibody. Sections were incubated overnight at $4{ }^{\circ} \mathrm{C}$ with polyclonal antibodies for V1a (SAB2501519, Sigma-Aldrich), V1b (HPA017337, Sigma-Aldrich) and V2 (sc-18101, Santa Cruz Biotechnology). The following day, samples were rinsed with PBS, incubated with secondary biotinylated antibody (LSAB Plus System; Dako, Milan, Italy), then with Dako ABC (LSAB Plus system), and finally developed with 3,3'-diaminobenzidine. To confirm the specificity of immunoreaction, negative controls were performed for all immunoreactions. Sections were examined with a Leica Microsystems DM 4500 B Microscopy equipped with a JenoptikProg Res C10 Plus Videocam. Observations were processed with an Image Analysis System (IAS; Delta Sistemi) and were independently performed by two researchers in a blinded manner.

\section{Immunofluorescence}

In vitro studies were performed using the mouse and human cell lines and normal and BDL rat cholangiocytes isolated from the experimental samples. We also used our immortalized murine cell lines of small and large cholangiocytes. ${ }^{26,27}$ The cells were maintained in a specific medium. A human immortalized non-malignant cholangiocyte cell line (H69, a gift of Dr Gregory Gores, Mayo Clinic, Rochester, MN, USA) and an immortalized cell line obtained from the epithelium lining the hepatic cysts from patients with ADPKD (LCDE or CL3, a gift of Dr Douglas Jefferson, Tufts Medical School, Boston, MA, USA) ${ }^{16}$ were also used. Cholangiocytes from cell lines were seeded on coverslip in a six-well plate and allowed to adhere overnight. Cells were fixed in $4 \%$ paraformaldehyde, and following washes and incubation in $4 \%$ bovine serum albumin (BSA) and PBS-T, they were incubated with 
the selected primary antibody (V1a, V1b, V2). Then the cells were washed in PBS-T and then placed in the specific Alexa Fluor 488 secondary antibody in a dark room. Finally, cholangiocytes were rinsed and the coverslip was put onto slide with a drop of DAPI.

\section{Real-Time PCR}

To evaluate the messenger expression of $\mathrm{V} 2$ in purified mouse (normal and BDL) and human (normal and ADPKD) cholangiocytes, we used the $\mathrm{RT}^{2}$ Real-Time assay from SABiosciences as described. ${ }^{26} \mathrm{~A} \Delta \Delta \mathrm{C}_{\mathrm{T}}$ (delta delta of the threshold cycle) analysis was performed using normal cholangiocytes as the control sample. The primer for V2 (purchased from SABiosciences) was designed according to the NCBI GenBank Accession number NM_001146151.1. ${ }^{1}$ Data were expressed as relative mRNA levels \pm s.e.

\section{Proliferation Assay and CAMP Levels}

H69 and LCDE following culture in the appropriate medium were deprived of serum for $24 \mathrm{~h}$. Cells were then maintained in serum-deprived conditions for an additional $24 \mathrm{~h}$ for MTT proliferation assay (controls) or exposed to serum, AVP (from 0.1 to $100 \mu \mathrm{g} / \mathrm{ml}$ ). Except for large mouse cholangiocytes, we also performed preincubation with or without Tolvaptan $(100 \mu \mathrm{g}),{ }^{28} \mathrm{a}$ V1a, V1b or V2 antagonist or OPC-31260, a specific V2 antagonist $(100 \mu \mathrm{g}) .{ }^{29}$ In detail, cell medium was replaced with a fresh serum-free medium with the tested agent. We used a commercially available colorimetric cell proliferation assay (CellTiter 96 aqueous nonradioactive cell proliferation assay, MTT Kit), following the manufacturer's instructions. Proliferation index was calculated as the ratio between cell numbers in both unstimulated and stimulated cultures.

The levels of cAMP, a functional parameter of cholangiocyte growth, ${ }^{30}$ were measured using EIA kits, purchased from Cayman Chemical. cAMP was determined in supernatants from small and large mouse cholangiocytes, H69 or LCDE treated with/without AVP in absence/presence of the two inhibitors (Tolvaptan and OPC-31260) according to the manufacture's protocol.

\section{Statistical Analysis}

Data are presented as arithmetic mean \pm s.d. Student's $t$-test or Mann-Whitney $U$-test was used to determine differences between groups for normally or not normally distributed data, respectively. A $P$-value of $<0.05$ was considered statistically significant. Statistical analyses were performed using the SPSS statistical software.

\section{RESULTS}

\section{Cholangiocytes Express the V2 Receptor}

Rat

Immunohistochemistry performed on the rat livers showed that $\mathrm{V} 1 \mathrm{a}$ and $\mathrm{V} 1 \mathrm{~b}$ expression in cholangiocytes is negligible in both normal and BLD rats, whereas V2 receptor is expressed in normal rat cholangiocytes and markedly expressed in BDL rat cholangiocytes (Figure 1a). Similarly, immunofluorescence confirmed that rat cholangiocytes express V2 in both cell lines, with the latter having an increased expression of the receptor. V1a and V1b were not expressed in both cell types (Figure 2a).

\section{Mouse}

Immunohistochemistry performed in the normal mouse livers showed that normal small and large cholangiocytes express V2 receptor, whereas V1a and V1b were not present. In BDL mice, the expression of V2 was significantly increased, whereas V1a and V1b expression remained negligible in BDL mice (Figure 1b). In vitro, small and large mouse cholangiocytes express V2 receptor. V1a and V1b are not expressed both in small and large cholangiocytes (Figure $2 \mathrm{~b}$ ). V2 receptor expression in cholangiocytes was confirmed by analyzing gene expression for this receptor in isolated cholangiocytes from both Sham and BDL mice. The latter were shown to have a doubled V2 receptor expression with respect to the Sham mice by real-time PCR (Figure 3a).

\section{Human}

The results obtained in mice and rats were confirmed in human samples. V1a and V1b were not expressed in the normal livers as well as hepatic cysts obtained from ADPKD patients, except for a slight positivity of V1b in the cystic epithelium. On the other hand, V2 was expressed by cholangiocytes in the normal livers; its expression was significantly increased in cholangiocytes lining hepatic cysts obtained from ADPKD patients (Figure 1c). In vitro, in human $\mathrm{H} 69$ cells the V2 receptor was shown to be expressed, whereas V1a and V1b were not present (Figure 2c). In LCDE cells, the expression of V1a was negative and V1b was weakly represented, whereas V2 was significantly increased above its normal expression level. In addition, V2 receptor gene expression was confirmed both in H69 and LCDE cells and was higher in LCDE cells when compared with H69 (Figure 3b).

\section{Measurement of Cholangiocyte Proliferation Mouse}

To verify whether vasopressin has a different role in mature and immature cholangiocytes, we studied its effects on small and large cholangiocyte growth. The MTT proliferation assay yielded different results in mouse small and large cholangiocytes. By increasing the amount of vasopressin provided to the small cholangiocyte cell lines, a concomitant proliferation was observed. At $0.1 \mu \mathrm{g}$, proliferation was 1.5 the normal, it doubled at $1 \mu \mathrm{g}$, then increased to 2.5 times than normal at $10 \mu \mathrm{g}$, and tripled at $100 \mu \mathrm{g}$. No effects on proliferation were observed in large cholangiocytes treated with vasopressin (Figure 4a). The administration of Tolvaptan or OPC-31260, V2 antagonists, ${ }^{31,32}$ to small cholangiocytes decreased the proliferative rate of these cells (Figure 4b). 
a
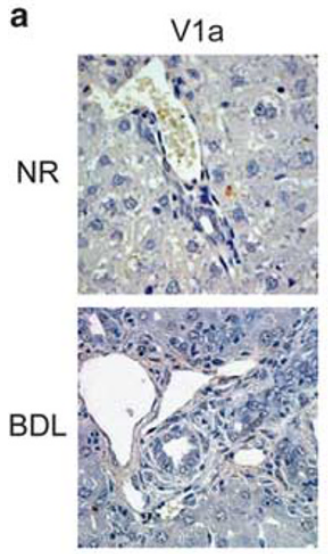

V1b
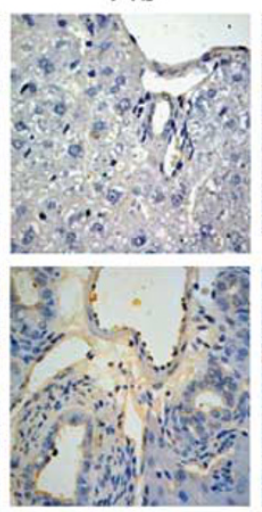
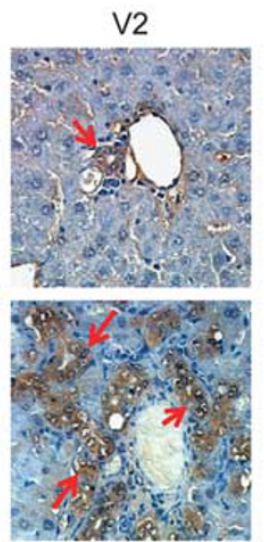

b
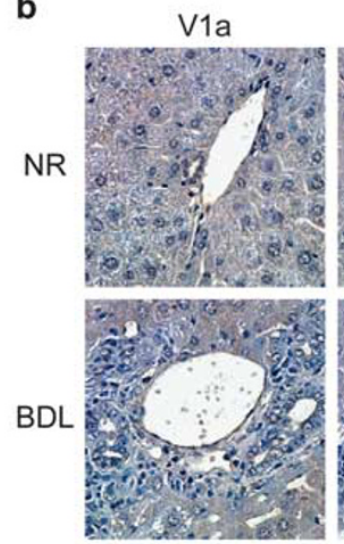
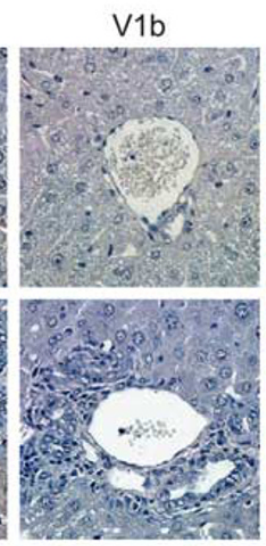
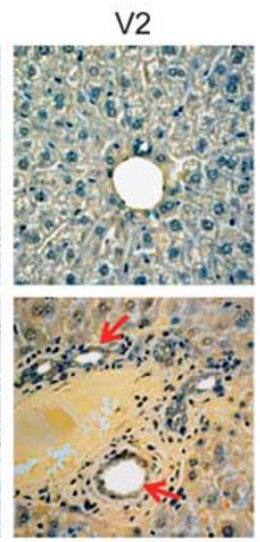

c
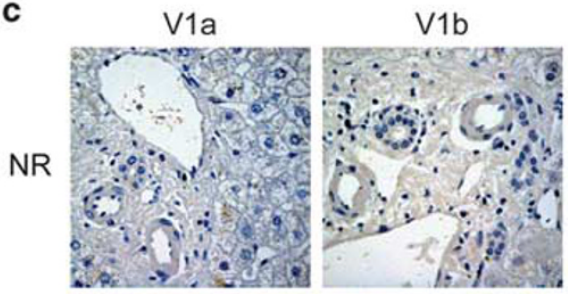

V2
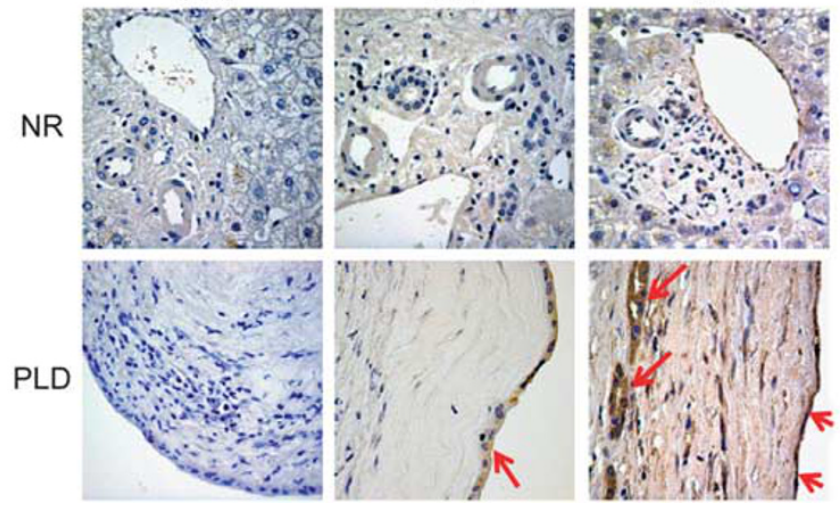

Figure 1 Representative immunohistochemistry for the three AVP receptors: V1a, V1b, and V2 in liver sections from Normal and BDL-1week rats (a), from Normal and BDL-1week mice (b), and from Normal and PLD human samples (c). Bile ducts express V2 subtype of vasopressin receptors in Normal animals and, with higher levels, in the experimental model of BDL, both in mouse and rat. In human samples, V2 is present in normal biliary epithelium and increases during PLD, both in small (diameter $<3 \mathrm{~mm}$ ) and large (diameter $>3 \mathrm{~mm}$ ) cysts. No or very low staining was visible in the immunohistochemistry for V1a and V1b. No staining was visible when primary antibodies were replaced with non-immune serum, original magnification, $\times 40$.

\section{Human}

MTT proliferation assay in $\mathrm{H} 69$ also yielded a slight increase in proliferation up to 1.01 at $1 \mu \mathrm{g}, 1.02$ at $10 \mu \mathrm{g}$ and 1.03 at $100 \mu \mathrm{g}$. Proliferation was not observed upon administering $100 \mu \mathrm{g}$ vasopressin in combination with Tolvaptan or $100 \mu \mathrm{g}$ vasopressin with OPC-31260 (Figure 4c). The same results were seen in LCDE cells, but the effect of vasopressin on proliferation was much more striking: at $1 \mu \mathrm{g}$ vasopressin proliferation was higher compared with the corresponding H69 value. Instead, when administering $100 \mu \mathrm{g}$ vasopressin with Tolvaptan or $100 \mu \mathrm{g}$ vasopressin with OPC-31260, there was no proliferation detectable (Figure 4c).

\section{Measurement of Intracellular Levels of cAMP}

Similar to that shown for secretin, we found in small mouse cholangiocytes that vasopressin increases cAMP levels, an increase that was prevented by preincubation with Tolvaptan or OPC-31260 (Figure 5a). Likewise, we have evaluated the effect of vasopressin and its receptor antagonists in human cell lines, and the intracellular vasopressin-stimulated cAMP levels as well as cholangiocyte proliferation increased, an enhancement blocked by preincubation with the two V2 receptor antagonists, Tolvaptan and OPC-31260 (Figure 5b). This supports the concept that vasopressin, both in mouse and human, sustains biliary growth via a cAMP-dependent signaling pathway.

\section{DISCUSSION}

The present study provides the following new findings: (i) mouse, rat and human cholangiocytes express V2 receptors that are upregulated following BDL as well as in cholangiocytes lining liver cysts in ADPKD patients; (ii) in vitro, the administration of vasopressin increased proliferation and cAMP levels of small mouse cholangiocytes and human LCDE cells, these effects were blocked by preincubation with Tolvaptan as well as OPC-31260, a specific V2 antagonist; and (iii) vasopressin does not induce significant changes in proliferation of large mouse cholangiocytes.

Cholangiocytes are characterized by marked proliferative capacities that are evident under different experimental 
conditions, such as BDL in mouse or rat models. ${ }^{17,33,34}$ These conditions mimic human chronic liver diseases, including primary biliary cirrhosis and polycystic liver disease, where the cholangiocytes represent the target of the disease. ${ }^{35,36}$ In particular, mutations in polycystins (PC1 or PC2) are a cause of polycystic liver disease, characterized by the progressive a
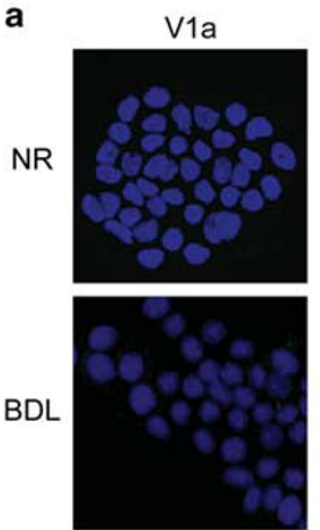

V1b
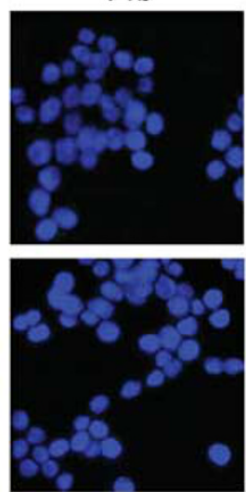

V2
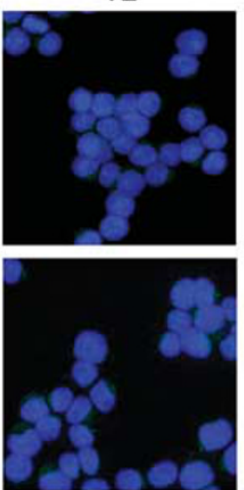

b
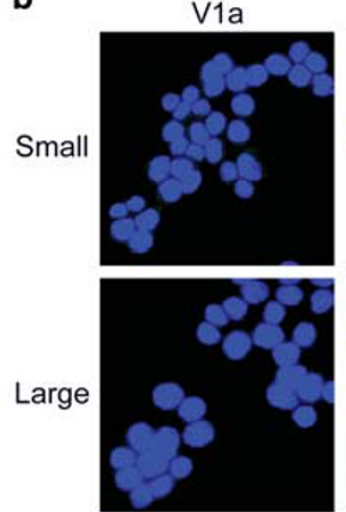

$\mathrm{V} 1 \mathrm{~b}$
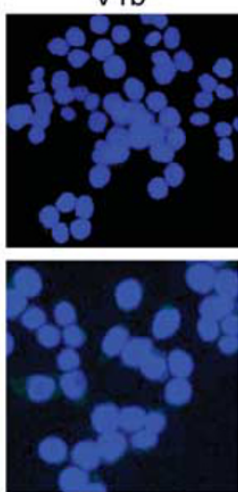

V2
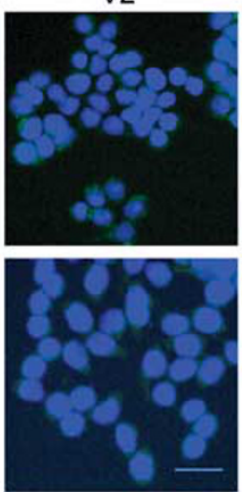

C
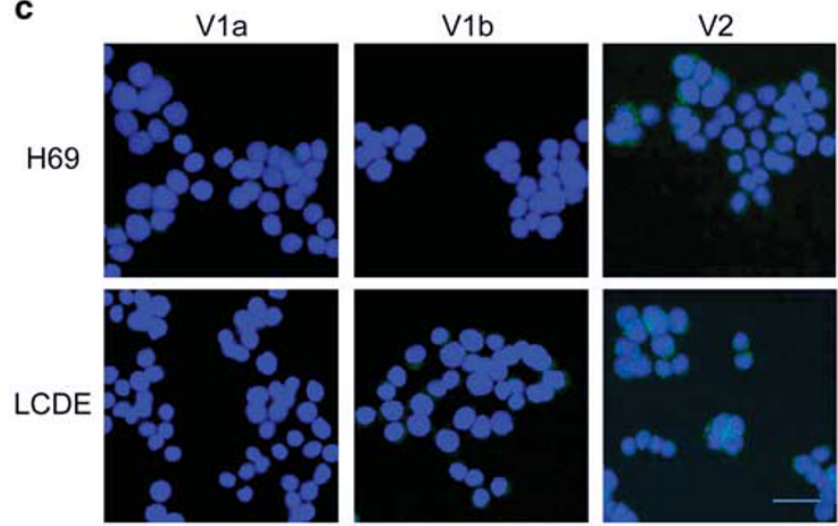

Figure 2 By immunofluorescence, cholangiocytes from Normal and BDL rats (a) express V2 receptor subtype. After BDL, cells present low expression of V1a and V1b but higher immunopositivity for V2. The same experiments were performed in small and large cholangiocytes from mice (b), where the expression of V2 is predominant both in small and large cells. Finally, in human cholangiocytes (c), the immunofluorescence demonstrated that they express V2 with greater amount in LCDE, the cells from the cystic epithelium. Specific immunoreactivity of representative fields is shown in green; cell nuclei were stained with DAPI (blue). No staining was visible when primary antibodies were replaced with non-immune serum (data not shown). $\operatorname{Bar}=200 \mu \mathrm{m}$.
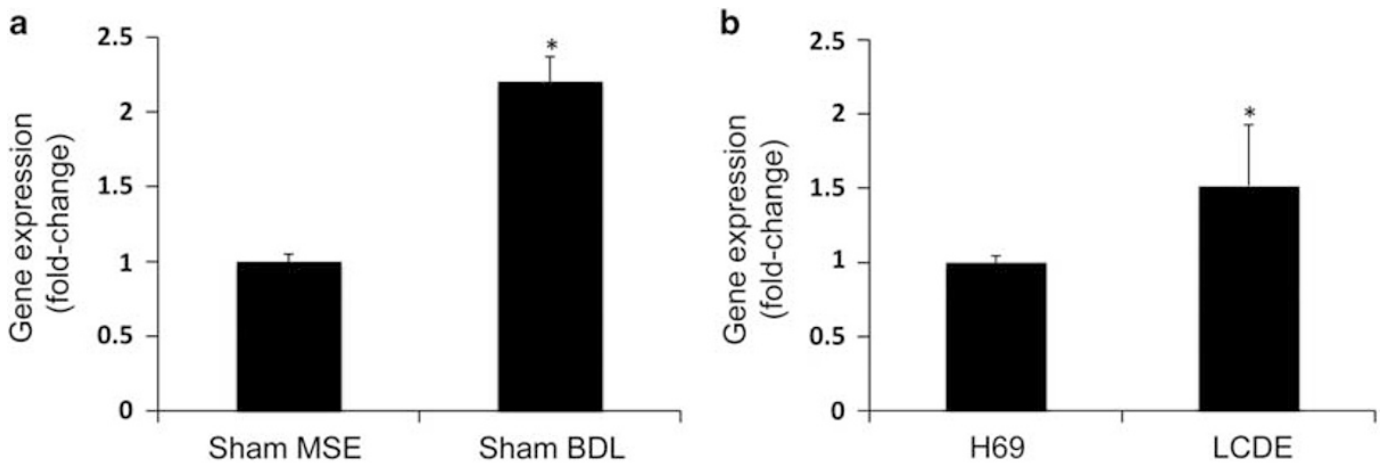

Figure 3 Evaluation of the mRNA by real-time PCR in purified cholangiocytes from (1) normal and BDL-1 week mouse; ${ }^{*} P<0.05$ vs sham cells (a) and (2) $\mathrm{H} 69$ and $\mathrm{LCDE} ;{ }^{*} \mathrm{P}<0.05$ vs H69 cells (b). Murine and human cholangiocytes express V2 mRNA. The expression of V2 was significantly increased in cholangiocytes from BDL mice compared with normal mice (a). As well as in LCDE cells compared with H69 cells (b). Data are mean \pm s.e.m. of three PCR reactions. 
a

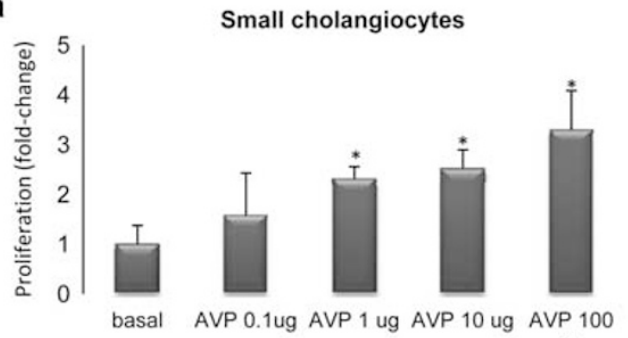

ug

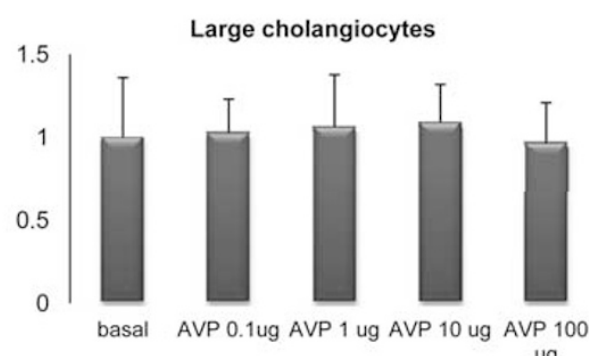

ug
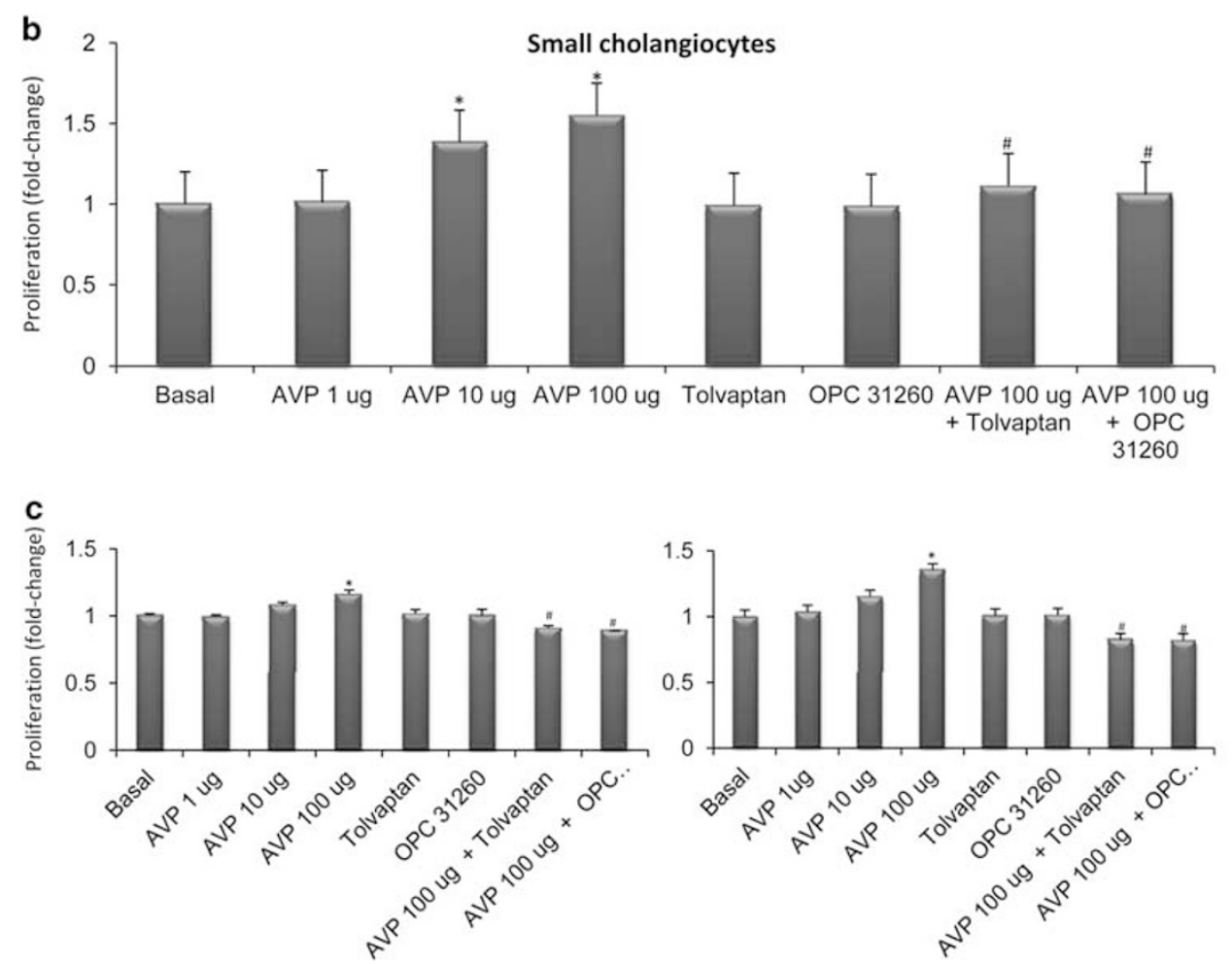

Figure 4 Evaluation of cholangiocyte proliferation with the administration of vasopressin $(0.1-100 \mu \mathrm{g} / \mathrm{ml})$ at different concentrations for $24 \mathrm{~h}$ by $\mathrm{MTT}$ assay in mice small and large cholangiocytes (a); in small cholangiocytes with the preincubation with two V2 antagonists: Tolvaptan and OPC-31260 (b); and in $\mathrm{H} 69$ and LCDE (c). Vasopressin increased growth of small cholangiocytes, H69 and LCDE in a dose-dependent manner, while large cholangiocytes are not sensitive to these concentrations of the hormone. The proliferation was also partly inhibited by the two V2 antagonists. ${ }^{*} P<0.05$ vs basal treatment, $\# P<0.05$ vs AVP $100 \mu \mathrm{g}$ treatment. Data are means \pm s.e. of five experiments.

development of multiple cysts that initially bud from the biliary epithelium with progressive lack of communication with the biliary tree. ${ }^{20,37} \mathrm{~A}$ higher production of cAMP represents the main signaling abnormality of cystic cholangiocytes that is responsible for the proliferative activity of cystic epithelium. ${ }^{38}$ In fact, studies in PC2-defective cholangiocytes have shown that both ERK1/2 and mTOR converge in stimulating cyclins and HIF $1 \alpha$-dependent VEGF-A secretion. ${ }^{39}$

The hormone vasopressin acts on different receptors, such as V1a, V1b and V2, but the binding with V2 is the main hormonal regulator of adenylyl cyclase activity in freshly dissected collecting ducts. ${ }^{40}$ The V2-induced effects on cAMP and water permeability may be limited by the action of AVP on V1a receptors stimulating phospholipase $\mathrm{C}$, phosphoinositide hydrolysis and $\mathrm{Ca}^{2+}$ release from the endoplasmic reticulum. ${ }^{41,42}$ Recent experimental studies have suggested a central role for vasopressin and $3^{\prime}-5^{\prime}$-cyclic adenosine monophosphate in promoting cyst growth, kidney enlargement and renal function decline in ADPKD. ${ }^{43}$ Inhibition of vasopressin by either a pharmacological agent such as vasopressin V2 receptor antagonist (V2RA) ${ }^{44,45}$ or drinking more water led to reduced growth of cysts and renal function preservation in animal models of polycystic kidney disease. ${ }^{46}$ In turn, the increased production of cAMP stimulates proliferation and growth of ADPKD cells and drives $\mathrm{Cl}^{-}$ and fluid secretion via protein kinase A-stimulated CFTR. ${ }^{47}$ Furthermore, the arginine vasopressin V2 receptor antagonists OPC-31260 and Tolvaptan inhibit the development of polycystic kidney disease in cpk mice and in three animal orthologs to human autosomal-recessive polycystic kidney 
a
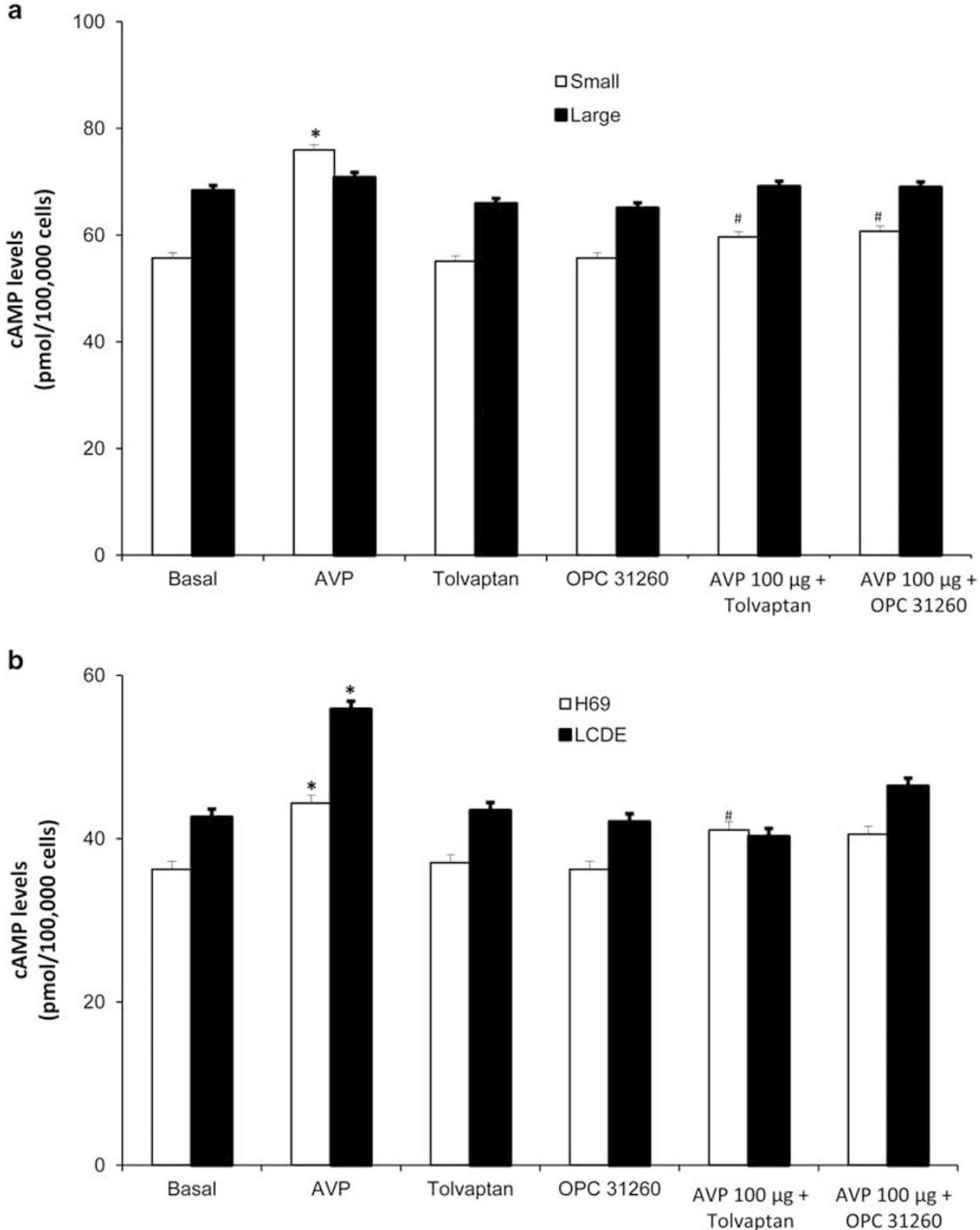

Figure 5 Intracellular cAMP levels were measured in: (a) small (white bar) and large cholangiocytes (black bar) and (b) also in H69 (white bar) and in LCDE (black bar) treated with $0.2 \%$ BSA (basal) vasopressin $(100 \mu \mathrm{g} / \mathrm{ml})$ in the absence or presence of Tolvaptan $(100 \mu \mathrm{g})$ or OPC-31260 $(100 \mu \mathrm{g})$. Vasopressin significantly increased the intracellular levels of CAMP mainly in small cholangiocytes, H69 and LCDE. Effects were blocked by preincubation with the two inhibitors, Tolvaptan and OPC-31260. Data are expressed as mean \pm s.d. of six experiments. ${ }^{*} P<0.05$ vs its corresponding basal value, $\# P<0.05$ vs its corresponding vasopressin treatment.

disease (PCK rat), autosomal-dominant polycystic kidney disease (Pkd2-/WS25 mice) and nephronophthisis (pcy mouse) by downregulating cAMP signaling, cell proliferation and chloride-driven fluid secretion. ${ }^{44,45,48,49}$ Instead, the V2 receptor agonist 1-deamino-8-d-arginine vasopressin increased renal cAMP and recovered the full cystic phenotype and aggravated the renal cystic disease. ${ }^{45,50}$ Our study has demonstrated that the receptor V2 is also expressed in cholangiocytes and this expression increases in the experimental model of mouse and rat BDL and in the human biliary epithelium that lines the hepatic cysts. In addition, the in vitro data have showed a proproliferative effect of vasopressin in small cholangiocytes, which represents the immature biliary compartment, activated to compensate for the damage of large cholangiocyte. ${ }^{51}$ In vitro, small and large mouse cholangiocytes express V2 receptor, while V1a and V1b are not expressed both in small and large cholangiocytes. Subsequently, we have analyzed cholangiocyte proliferation after AVP treatment in the same types of cells by MTT to demonstrate that small cholangiocytes significantly enhance 
their growth with vasopressin administration, whereas large cells display no significant increase. The reason could be linked, even if we did not perform analysis for that, to the quantity of receptor (V2) expressed by the two different types of cholangiocytes. Probably small cholangiocytes contain a higher presence of $\mathrm{V} 2$ receptor that lead to increase more the activation and production of cAMP. Instead, large cells may express a small amount of receptors to partially block the possibility to proliferate. Moreover, AVP enhances the growth of the hepatic cystic epithelium by the activation of the cAMP signaling pathway. These specific effects were blocked if we used Tolvaptan and OPC-31260, which selectively antagonizes V2 receptors, with a reduced proliferation and a decrease in cAMP levels. In fact, AVP exerts its effect by binding to three types of receptors: V1a, V1b, and V2. These receptors are G-protein-coupled with seven transmembrane domains, but their roles, activation and regulation of mRNA expression are very different. In a recent study of Tran et al, it is reported that AVP increases intracellular $\mathrm{Ca}^{2+}$ in human adipose-derived stromal/stem cells by activating V1a receptors and the $\mathrm{PLC}-\mathrm{IP}_{3}$ pathway. In contrast, mouse embryonic stem cells express all three receptor subtypes with different expression levels. ${ }^{52}$ Moreover, Gassanov's group showed that AVP facilitates cardiomyogenesis by activating NO signaling via V2 receptor; however $\mathrm{Ca}^{2+}$ signaling was not discovered. Whereas AVP, via the type 2 receptor (V2), activates the cAMP/PKA/cAMP response element-binding protein pathway. ${ }^{53}$ So the different expression and link with a receptor can lead to a different activation pathway in several type of cells. In our case, cholangiocytes express V2 and a cAMPdependent pathway is activated. Normally, large cholangiocytes are responsible for the majority of biliary fluid secretion, through the activation of a cAMP-dependent pathway. In small cholangiocytes, on the other hand, $\mathrm{Ca}^{2+}$-activated signaling pathways seem predominant. ${ }^{26}$ Indeed, the activation of purinergic receptors in small and large cholangiocytes induces $\mathrm{Ca}^{2+}$-dependent $\mathrm{Cl}^{-}$secretion via TMEM16A, providing an alternative route to the secretin-stimulated cAMP-dependent ductal fluid secretion. For these reasons, we can speculate that, as small cholangiocytes express only $\mathrm{V} 2$, the link of AVP with the receptor leads to the activation of a cAMP-dependent signaling pathway in these immature cells but that the stimulation with exogenous AVP may induce and start a quick transformation in mature cholangiocytes.

In summary, the results of this study indicate that AVP is a modulator of cholangiocyte proliferation. Like in the kidney also in the liver, cyst epithelial cells are persistently stimulated to proliferate and secrete fluid; this may be further enhanced by the renal and hepatic concentrating defect and increased circulating levels of AVP that occur in PKD and PLD. These observations could provide further support for the management and the modulation of V2 receptor in the regulation of the proliferation rate of the biliary epithelium during PLD.

\section{ACKNOWLEDGMENTS}

This work was supported by the Dr Nicholas C. Hightower Centennial Chair of Gastroenterology from Baylor Scott \& White; a VA Research Career Scientist Award; NIH grant DK58411 to Dr Alpini and Dr Glaser; a VA Merit Award (5I01BX000574) to Dr Alpini; a VA Merit Award (5101BX002192) to Dr Glaser from the United States Department of Veterans Affairs Biomedical Laboratory Research; Development Service, by University of Rome "La Sapienza", to Dr Gaudio and P. Onori; and FIRB Accordi di Programma 2010 \#RBAP10Z7FS to Dr Gaudio. This manuscript is the result of work supported by resources at the Central Texas Veterans Health Care System. The views expressed in this article are those of the authors and do not necessarily represent the views of the Department of Veterans Affairs.

\section{DISCLOSURE/CONFLICT OF INTEREST}

The authors declare no conflict of interest.

1. Armstrong SP, Seeber RM, Ayoub MA, et al. Characterization of three vasopressin receptor 2 variants: an apparent polymorphism (V266A) and two loss-of-function mutations (R181C and M311V). PLoS One 2013;8:e65885.

2. Serriere V, Tran D, Stelly N, et al. Vasopressin-induced morphological changes in polarized rat hepatocyte multiplets: dual calciumdependent effects. Cell Calcium 2008;43:95-104.

3. Nicou A, Serriere V, Prigent $S$, et al. Hypothalamic vasopressin release and hepatocyte $\mathrm{Ca} 2+$ signaling during liver regeneration: an interplay stimulating liver growth and bile flow. FASEB J 2003;17:1901-1903.

4. Koshimizu TA, Nakamura K, Egashira N, et al. Vasopressin V1a and V1b receptors: from molecules to physiological systems. Physiol Rev 2012;92:1813-1864.

5. Serriere V, Berthon B, Boucherie S, et al. Vasopressin receptor distribution in the liver controls calcium wave propagation and bile flow. FASEB J 2001;15:1484-1486.

6. Morel A, O'Carroll AM, Brownstein MJ, et al. Molecular cloning and expression of a rat V1a arginine vasopressin receptor. Nature 1992;356: 523-526.

7. Sugimoto $T$, Saito $M$, Mochizuki $S$, et al. Molecular cloning and functional expression of a cDNA encoding the human $\mathrm{V} 1 \mathrm{~b}$ vasopressin receptor. J Biol Chem 1994;269:27088-27092.

8. Grazzini E, Breton C, Derick $S$, et al. Vasopressin receptors in human adrenal medulla and pheochromocytoma. J Clin Endocrinol Metab 1999;84:2195-2203.

9. Lolait SJ, O'Carroll AM, McBride OW, et al. Cloning and characterization of a vasopressin V2 receptor and possible link to nephrogenic diabetes insipidus. Nature 1992;357:336-339.

10. Gieldon A, Kazmierkiewicz R, Slusarz R, et al. Molecular modeling of interactions of the non-peptide antagonist YM087 with the human vasopressin V1a, V2 receptors and with oxytocin receptors. J Comput Aided Mol Des 2001;15:1085-1104.

11. Goel M, Zuo CD, Schilling WP. Role of CAMP/PKA signaling cascade in vasopressin-induced trafficking of TRPC3 channels in principal cells of the collecting duct. Am J Physiol Renal Physiol 2010;298:F988-F996.

12. Torres VE. Vasopressin antagonists in polycystic kidney disease. Kidney Int 2005;68:2405-2418.

13. Iannucci NB, Ripoll GV, Garona J, et al. Antiproliferative effect of 1-deamino-8-D-arginine vasopressin analogs on human breast cancer cells. Future Med Chem 2011;3:1987-1993.

14. Bankir L, Bouby N, Ritz E. Vasopressin: a novel target for the prevention and retardation of kidney disease? Nat Rev Nephrol 2013;9: 223-239.

15. Wills ES, Roepman R, Drenth JP. Polycystic liver disease: ductal plate malformation and the primary cilium. Trends Mol Med 2014;20: 261-270.

16. Onori $P$, Mancinelli $R$, Franchitto $A$, et al. Role of follicle-stimulating hormone on biliary cyst growth in autosomal dominant polycystic kidney disease. Liver Int 2013;33:914-925.

17. Glaser $\mathrm{S}$, Onori $\mathrm{P}$, Wise $\mathrm{C}$, et al. Recent advances in the regulation of cholangiocyte proliferation and function during extrahepatic cholestasis. Dig Liver Dis 2010;42:245-252.

18. Hildebrandt F, Benzing $T$, Katsanis N. Ciliopathies. N Engl J Med 2011;364:1533-1543. 
19. Masyuk $\mathrm{Al}$, Huang $\mathrm{BQ}$, Radtke $\mathrm{BN}$, et al. Ciliary subcellular localization of TGR5 determines the cholangiocyte functional response to bile acid signaling. Am J Physiol Gastrointest Liver Physiol 2013;304: G1013-G1024.

20. Onori P, Franchitto A, Mancinelli R, et al. Polycystic liver diseases. Dig Liver Dis 2010;42:261-271.

21. Gevers TJ, Drenth JP. Somatostatin analogues for treatment of polycystic liver disease. Curr Opin Gastroenterol 2011;27:294-300.

22. Gevers TJ, Drenth JP. Diagnosis and management of polycystic liver disease. Nat Rev Gastroenterol Hepatol 2013;10:101-108.

23. Masyuk TV, Masyuk Al, Torres VE, et al. Octreotide inhibits hepatic cystogenesis in a rodent model of polycystic liver disease by reducing cholangiocyte adenosine 3',5'-cyclic monophosphate. Gastroenterology 2007;132:1104-1116.

24. Devuyst O, Torres VE. Osmoregulation, vasopressin, and CAMP signaling in autosomal dominant polycystic kidney disease. Curr Opin Nephrol Hypertens 2013;22:459-470.

25. Alpini G, Lenzi R, Sarkozi L, et al. Biliary physiology in rats with bile ductular cell hyperplasia. Evidence for a secretory function of proliferated bile ductules. J Clin Invest 1988;81:569-578.

26. Francis $\mathrm{H}$, Glaser $\mathrm{S}$, Demorrow $\mathrm{S}$, et al. Small mouse cholangiocytes proliferate in response to $\mathrm{H} 1$ histamine receptor stimulation by activation of the IP3/CaMK I/CREB pathway. Am J Physiol Cell Physiol 2008:295:C499-C513.

27. Ueno $Y$, Alpini G, Yahagi $K$, et al. Evaluation of differential gene expression by microarray analysis in small and large cholangiocytes isolated from normal mice. Liver Int 2003;23:449-459.

28. Reif $G A$, Yamaguchi $T$, Nivens $E$, et al. Tolvaptan inhibits ERKdependent cell proliferation, $\mathrm{Cl}(-)$ secretion, and in vitro cyst growth of human ADPKD cells stimulated by vasopressin. Am J Physiol Renal Physiol 2011;301:F1005-F1013.

29. Meijer E, Gansevoort RT, de Jong PE, et al. Therapeutic potential of vasopressin V2 receptor antagonist in a mouse model for autosomal dominant polycystic kidney disease: optimal timing and dosing of the drug. Nephrol Dial Transplant 2011;26:2445-2453.

30. Alpini $G$, Glaser $S$, Ueno $Y$, et al. Heterogeneity of the proliferative capacity of rat cholangiocytes after bile duct ligation. Am J Physiol Gastrointest Liver Physiol 1998;274(Pt 1):G767-G775.

31. Kogiso $T$, Tokushige $K$, Hashimoto $E$, et al. Safety and efficacy of longterm tolvaptan therapy for decompensated liver cirrhosis. Hepatol Res 2015;46:E194-E200.

32. Higashijima $\mathrm{Y}$, Sonoda $\mathrm{H}$, Takahashi $\mathrm{S}$, et al. Excretion of urinary exosomal AQP2 in rats is regulated by vasopressin and urinary $\mathrm{pH}$. Am J Physiol Renal Physiol 2013;305:F1412-F1421.

33. Glaser SS, Gaudio E, Miller T, et al. Cholangiocyte proliferation and liver fibrosis. Expert Rev Mol Med 2009;11:e7.

34. Munshi MK, Priester S, Gaudio E, et al. Regulation of biliary proliferation by neuroendocrine factors: implications for the pathogenesis of cholestatic liver diseases. Am J Pathol 2011;178:472-484.

35. Alvaro $D$, Onori $P$, Alpini $G$, et al. Morphological and functional features of hepatic cyst epithelium in autosomal dominant polycystic kidney disease. Am J Pathol 2008;172:321-332.
36. Franchitto $A$, Onori $\mathrm{P}$, Renzi $\mathrm{A}$, et al. Recent advances on the mechanisms regulating cholangiocyte proliferation and the significance of the neuroendocrine regulation of cholangiocyte pathophysiology. Ann Transl Med 2013;1:27.

37. Everson GT, Helmke SM, Doctor B. Advances in management of polycystic liver disease. Expert Rev Gastroenterol Hepatol 2008;2: 563-576.

38. Spirli C, Morell CM, Locatelli L, et al. Cyclic AMP/PKA-dependent paradoxical activation of Raf/MEK/ERK signaling in polycystin-2 defective mice treated with sorafenib. Hepatology 2012;56:2363-2374.

39. Spirli C, Okolicsanyi S, Fiorotto R, et al. Mammalian target of rapamycin regulates vascular endothelial growth factor-dependent liver cyst growth in polycystin-2-defective mice. Hepatology 2010;51:1778-1788.

40. Yasuda G, Jeffries WB. Regulation of cAMP production in initial and terminal inner medullary collecting ducts. Kidney Int 1998;54:80-86.

41. Bankir L. Antidiuretic action of vasopressin: quantitative aspects and interaction between V1a and V2 receptor-mediated effects. Cardiovasc Res 2001;51:372-390.

42. Torres VE. Vasopressin antagonists in polycystic kidney disease. Semin Nephrol 2008;28:306-317.

43. Belibi FA, Reif G, Wallace DP, et al. Cyclic AMP promotes growth and secretion in human polycystic kidney epithelial cells. Kidney Int 2004;66:964-973.

44. Gattone 2nd VH, Wang X, Harris PC, et al. Inhibition of renal cystic disease development and progression by a vasopressin V2 receptor antagonist. Nat Med 2003;9:1323-1326.

45. Wang X, Gattone 2nd V, Harris PC, et al. Effectiveness of vasopressin V2 receptor antagonists OPC-31260 and OPC-41061 on polycystic kidney disease development in the PCK rat. J Am Soc Nephrol 2005;16: 846-851.

46. Nagao S, Nishii K, Katsuyama M, et al. Increased water intake decreases progression of polycystic kidney disease in the PCK rat. J Am Soc Nephrol 2006;17:2220-2227.

47. Terryn S, Ho A, Beauwens R, et al. Fluid transport and cystogenesis in autosomal dominant polycystic kidney disease. Biochim Biophys Acta 2011;1812:1314-1321.

48. Torres VE. Role of vasopressin antagonists. Clin J Am Soc Nephrol 2008;3:1212-1218.

49. Torres VE, Harris PC, Pirson Y. Autosomal dominant polycystic kidney disease. Lancet 2007;369:1287-1301.

50. Wang $\mathrm{X}, \mathrm{Wu} \mathrm{Y}$, Ward $\mathrm{CJ}$, et al. Vasopressin directly regulates cyst growth in polycystic kidney disease. J Am Soc Nephrol 2008;19: 102-108.

51. Mancinelli R, Franchitto A, Glaser $\mathrm{S}$, et al. GABA induces the differentiation of small into large cholangiocytes by activation of $\mathrm{Ca}(2+) / \mathrm{CaMK}$ I-dependent adenylyl cyclase 8. Hepatology 2013;58:251-263.

52. Tran TD, Gimble JM, Cheng H. Vasopressin-induced Ca (2+) signals in human adipose-derived stem cells. Cell Calcium 2016;59: 135-139.

53. Gassanov N, Jankowski M, Danalache B, et al. Arginine vasopressinmediated cardiac differentiation: insights into the role of its receptors and nitric oxid signaling. J Biol Chem 2007;282:11255-11265. 